\title{
Distortion-Optimal Transmission of Progressive Images over Channels with Random Bit Errors and Packet Erasures
}

\author{
Homayoun Yousefi'zadeh Hamid Jafarkhani Farzad Etemadi \\ Department of EECS \\ University of California, Irvine
}

[hyousefi, hamidj, fetemadi] @uci.edu

\begin{abstract}
We present a statistical optimization framework for solving the end-to-end problem of progressive transmission of images over noisy channels. We consider the impacts of transmission bit errors as well as packet erasures. To cope with the impact of random bit errors, we formulate an optimization problem aimed at minimizing the end-to-end expected distortion of a reconstructed image subject to rate and efficiency constraints. In order to eliminate the impact of packet erasures, we propose utilizing an algorithm that is capable of statistically guaranteeing the delivery of a packet set associated with the progressive bitstream of an image source. Using receiver feedback, our framework is capable of effectively coping with the channel loss effects characterized by the Gilbert-Elliott model.
\end{abstract}

\section{Index Terms}

Progressive Transmission of Images, Source Coding, Channel Coding, Random Bit Error, Packet Erasure, Gilbert-Elliott Loss Model, Statistical Guarantee of Packet Delivery.

\section{INTRODUCTION}

Progressive transmission of images has proven as a viable alternative of delivering images over noisy channels. A progressive source coder provides the decoder with the capability of reconstructing the source data at different bit rates from the prefixes of a single bitstream. Due to high sensitivity to transmission noise, progressive transmission of images over noisy channels has to be accompanied with appropriate channel coding or joint source-channel coding schemes. A review of the literature reveals a rich set of articles within the context of progressive transmission of images over noisy channels. In the area of source coding, the works of Shapiro [16] introducing embedded zerotrees of wavelets, Said et al. [14] proposing set partitioning in hierarchical trees (SPIHT), Taubman et al. [21] suggesting a progressive wavelet-based subband image coding algorithm, Ordentlich et al. [13] covering embedded coding of the bitplanes of a wavelet-transformed image, and Malavar [11] suggesting yet another progressive wavelet coding technique are perhaps most closely related to our work. In the area of channel coding and joint source-channel coding, researchers have looked at two closely related but not exactly identical family of problems. These are namely minimizing distortion or distortion-optimal problems and maximizing useful source coding or rate-optimal problems. Rate-optimal problems have been proposed as lower complexity alternatives to distortion-optimal problems. Sherwood et al. [17] first proposed concatenating a source coder bitstream with an outer cyclic redundancy check (CRC) coder and an inner rate compatible punctured convolutional (RCPC) coder. Focusing on the rate-optimal problems and variable-length packets with fixed data payloads, Chande et al. in [6] and [5] proposed the use of dynamic programming and exhaustive search for protecting the source coder bitstream transmitted over Binary Symmetric Channels (BSC) and channels with memory, respectively. Banister et al. [2] proposed the use of a brute-force search algorithm to solve a distortion-optimal problem in a BSC to protect 
JPEG2000 coded images with an outer CRC coder and an inner punctured turbo coder. They also solved a sub-optimal problem with dynamic programming. Stankovic et al. [19] provided an algorithm that was capable of accelerating the computation of the optimal strategy of [6] for the case of fixed-length packets. Appadwedula et al. [1] relied on the exponential rate-distortion model of an image coder to analytically solve the distortion-optimal problem for a BSC. Lu et al. [10] solved a similar distortion-optimal problem relying on data fitting techniques for BSC's. When attempting at applying their approach to the case of channels with memory, their approach resulted in very conservative estimates of channel error probability. As an alternative to directly applying channel coding techniques in conjunction with the source coding techniques, Srinivas et al. [18] proposed utilizing a maximum a posteriori (MAP) detector to compensate for the impacts of spatially correlated compressed bitstream as well as temporally correlated channel errors. Their approach called for the utilization of interleaving techniques when dealing with temporally correlated channel errors. Without investigating the optimality of their approach, Cosman et al. [7] showed the potential advantage of using a hybrid technique of adding channel coding to wavelet-based zerotree encoded images and reordering the resulting embedded zerotree bitstream into packets with a small set of wavelet coefficient trees.

A review of the literature articles reveals that there has not been any systematic study of the subject material for the noisy channels with temporally correlated random bit errors and packet erasures. This paper proposes a statistical optimization framework for progressive transmission of images over such channels. The framework consists of two components the combination of which is capable of dealing with temporally correlated random bit errors and packet erasures. The random bit error component can be applied either as a distortion-optimal problem or as a rate-optimal problem. Further, it can be applied to both fixed-length and variable-length packet scenarios. The statistical packet erasure component consists of an algorithm that can guarantee the delivery of a block of packets with a given probability. The framework also proposes the use of feedback when integrating its components.

An outline of the paper follows. In Section II, we describe our integrated protocol. In Section III, we review the characteristics of our proposed channel coding technique. We also analyze the channel loss behavior relying on the Gilbert-Elliott model. In Section IV, we discuss the random bit error component of our optimization framework. Our discussion includes a probabilistic formulation of the optimization problem along with the solution to it. In Section V, we describe the packet erasure component of our framework. The proposed algorithm of this section is capable of statistically compensating for packet erasures. In Section VI, we numerically validate our results. Finally, Section VII includes a discussion of concluding remarks.

\section{Description of The End-TO-End Integrated Protocol}

In this section, we provide a description of our end-to-end integrated protocol. We consider the transmission of a bitstream produced by a progressive image source coder such as SPIHT [14] or JPEG2000 [20] over a noisy channel. We assume that the bitstream is packetized into a number of packets with a fixed or a variable number of source bits per packet. We consider errors associated with both bit errors and packet erasures. The utilization of error detection and error correction channel codes can potentially compensate for the error effects at both bit and packet levels. Encoding the information bits of individual packets, we note that each packet will contain source coding and/or channel coding (parity) bits. The calculation of parity bits is done based on minimizing the expected distortion of the reconstructed bitstream. Further, we propose the use of packet level channel coding to compensate for the packet erasure impacts on the packetized bitstream. Such a coding scheme treats the collection of information and parity bits in each packet as data for the purpose of packet 
erasure compensation. Our integrated protocol is categorized under type II hybrid Automatic Repeat reQuest (ARQ) and Forward Error Correction (FEC) protocols. In a type II hybrid protocol, a retransmission request is responded by transmitting a codeword containing extra parity bits for a previously transmitted codeword. Relying on the discussion of RS codes in [22] and [4] together with receiver feedback and erasure decoding, we propose the use of systematic rate compatible punctured RS codes in our protocol to compensate for both random bit errors and packet erasures. We note that the systematic rate compatible punctured RS codes together with erasure decoding outperform nonsystematic RS codes of [12]. Fig. 1 depicts the flowchart of our end-to-end protocol.

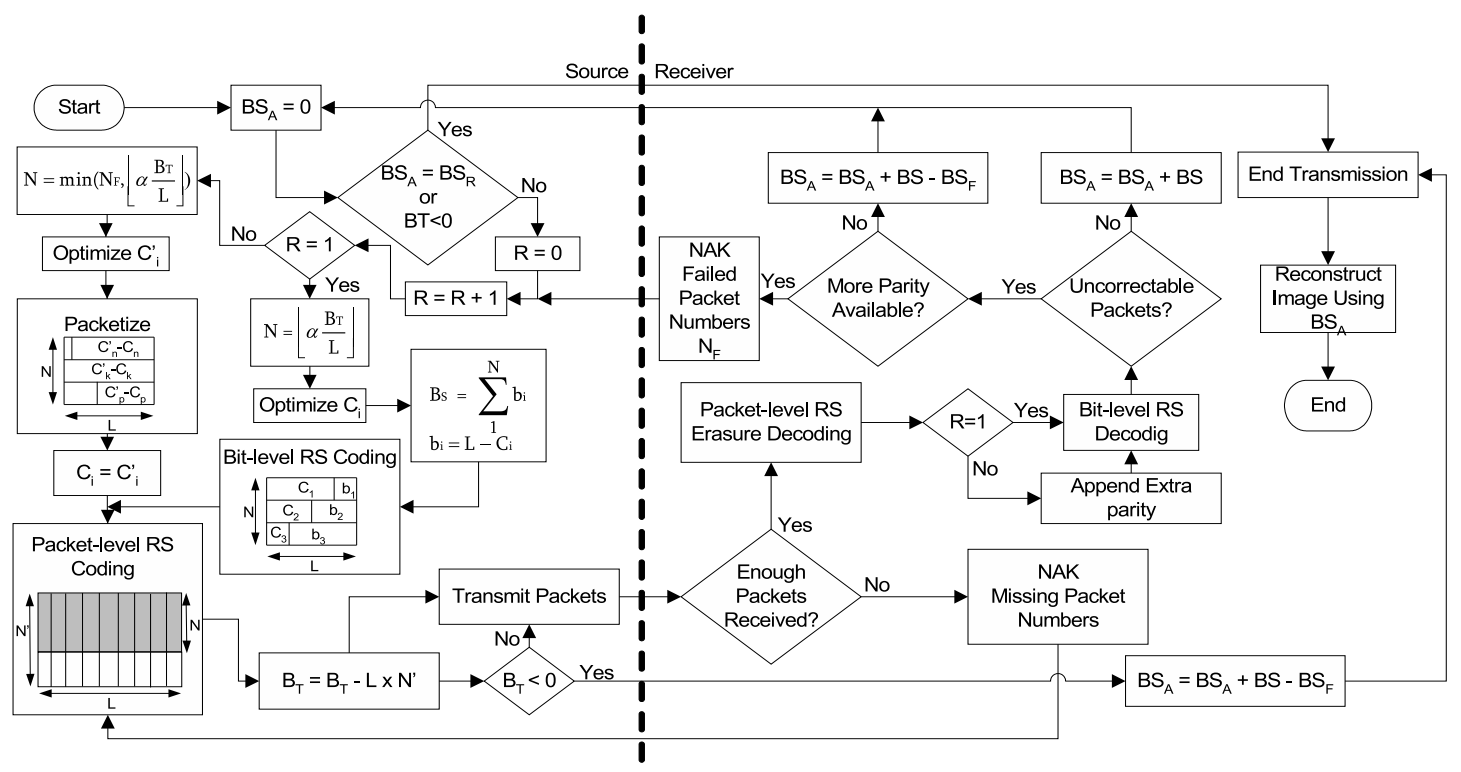

Fig. 1. The flowchart of the proposed end-to-end protocol.

We assume that a bit budget $B_{T}$ and a per round probability of delivering a packet set are given. Our round-based protocol consists of two components. The first component is used to compensate for the random bit errors. The second component is used to recover erased packets. $B S_{A}$ represents the accumulated transmitted bitstream in each round and never exceeds $B S_{R}$. Assuming a fixed packet length of $L$, the number of packets to transmit the bitstream is chosen as $N=\min \left(\left\lfloor\alpha \frac{B_{T}}{L}\right\rfloor,\left\lfloor\frac{B S_{R}-B S_{A}}{L}\right\rfloor\right)$. where $0<\alpha<1$ is a design parameter effectively splitting the available budget $B_{T}$ between the two components of the protocol. The optimal number of per packet parity symbols minimizing the expected distortion of the reconstructed image is then calculated according to the discussion of Section IV. Next, the total number of data and parity packets $N^{\prime}$ required to statistically guarantee the delivery of the packet set is calculated according to the discussion of Section V. After updating transmission budget $B_{T}$, announcing the start time and the duration of the round, the source proceeds with the transmission of the coded packet set to the receiver. Each packet includes a sequence number. The receiver waits for the duration of the round before determining whether it has received $N$ packets required to recover the packet set. At the end of the round, the receiver sends the packet numbers of the erased packets to the source in a single packet NAK message if it has not bot been able to recover the block of packets. The NAK message includes a two bit per packet bitmap associated with the individual packets of the set. The receiver sets the $M S B$ bit associated with a packet to zero if it has been able to recover the packet. With the $M S B$ bit set to one, the receiver sets the $L S B$ bit to one if it has not been able to recover the packet due to an erasure. We also note that utilization of similar error detection and correction 
codes along with the employment of timeout mechanisms can effectively cope with the impacts of random bit errors and packet erasures in the transmission of single packet NAK and control messages. For simplicity, we assume that the transmission of single packet NAK and control messages are error free in the rest of our discussion. The source then retransmits an extra number of packets in order to compensate for packet erasures in the channel. The number of extra packets is again calculated from the statistical guarantee algorithm of Section V. Once the receiver has recovered the block of packets, it aims at recovering the source coding bits in each packet. In the first round, each packet contains image data and parity symbols and is directly decoded to recover image data. In the second and later rounds, each packet includes incremental redundant symbols. The receiver thus needs to append them to the previously uncorrectable packets. In either case, the contents of the receiver buffer are decoded. If there are no uncorrectable packets and the collective number of source coding bits $B S_{A}$ is less than $B S_{R}$, another set of rounds is initiated starting from the first round. However, if uncorrectable packets exist, the receiver requests extra redundant symbols for those $N_{F}$ packets by setting per packet bits of those packets to $(M S B, L S B)=(1,0)$ in the NAK message. If uncorrectable packets exist but their RS code has reached the length of the mother code, the transmission of the current set is started from the first round after checking the available budget. Otherwise, another round of delivering extra redundant symbols initiates. The amount of redundancy is determined by increasing the error correcting capability of each packet of the first round to an amount calculated from the optimization algorithm of Section IV for the second round and beyond. Next, the source packetizes extra parity bits using appropriate header paddings and choosing $N=\min \left(N_{F},\left\lfloor\alpha \frac{B_{T}}{L}\right\rfloor\right)$. It will then transmits the packets to the receiver. The entire set of rounds and consequently the image transmission terminate under one of the two following conditions: (1) the entire bitstream $B S_{R}$ has been received with no uncorrectable blocks, and (2) the transmission budget is exhausted. The image is reconstructed using all of the packets preceding the first uncorrectable packet immediately after the transmission is terminated. We note that although the second termination scenarios involve distortion, a perfect image reconstruction is possible under the first scenario.

\section{Channel Coding And Loss Analysis}

In this section, we first describe our proposed channel coder properties and then continue by providing an analysis of the Gilbert-Elliot channel loss model of [8]. We note that a rate compatible punctured RS channel coder converts $k$ information bits (packets) into a sequence of $n_{i}$-bit ( $n_{i}$ packet) blocks where $i=1, \cdots, r$. For the first sequence, $\left(n_{1}-k\right)$ parity bits (packets) are appended to $k$ data bits (packets). The sequences $n_{j}$ for $j=2, \cdots, r$ are obtained by appending $n_{j}-n_{j-1}$ bits (packets) to the previous $n_{j-1}$ bits (packets). We also note that the combined channel code $n_{i}$ is capable of correcting up to $t_{c}=\left\lfloor\frac{n_{i}-k}{2}\right\rfloor$ bit (packet) errors.

In order to calculate the error rate of a block utilizing an $R S(n, k)$ coder, we consider the two-state Gilbert-Elliott loss model representing a channel with temporally correlated loss. In the Gilbert-Elliott model, bit (packet) loss is described by a two-state Markov chain. The GOOD state represents the loss of a bit (packet) with probability $\varepsilon_{G}$ while the BAD state represents the loss of a bit (packet) with probability $\varepsilon_{B}$ where $\varepsilon_{B}>>\varepsilon_{G}$. The GOOD state also introduces a probability $\gamma$ of staying in the GOOD state and a probability $1-\gamma$ of transitioning to the BAD state while the BAD state introduces a probability $\beta$ of staying in the BAD state and a probability $1-\beta$ of transitioning to the GOOD state. Typically, the parameters $\gamma$ and $\beta$ are measured from the observed loss rate and average burst length. In [9], we provide effective ways of measuring the parameters of the Gilbert-Elliott loss model. For the Gilbert-Elliott loss model, the probability of receiving exactly $k$ 
bits (packets) from $n$ transmitted bits (packets) is described as

$$
P(n, k)=P(n, k, G)+P(n, k, B)
$$

The recursive probabilities of receiving exactly $k$ bits (packets) from $n$ transmitted bits (packets) and winding up in the GOOD state and the BAD state are respectively given by

$$
\begin{aligned}
P(n, k, G) & =\varepsilon_{G}[\gamma P(n-1, k, G)+(1-\beta) P(n-1, k, B)] \\
& +\left(1-\varepsilon_{G}\right)[\gamma P(n-1, k-1, G)+(1-\beta) P(n-1, k-1, B)]
\end{aligned}
$$

and

$$
\begin{aligned}
P(n, k, B) & =\varepsilon_{B}[(1-\gamma) P(n-1, k, G)+\beta P(n-1, k, B)] \\
& +\left(1-\varepsilon_{B}\right)[(1-\gamma) P(n-1, k-1, G)+\beta P(n-1, k-1, B)]
\end{aligned}
$$

for $n \geq k>0$ and the initial conditions

$$
\begin{array}{llrl}
P(0,0, G) & =g_{s s}=\frac{1-\beta}{2-\gamma-\beta}, & & P(1,0, G)=\varepsilon_{G}\left[\gamma g_{s s}+(1-\beta) b_{s s}\right] \\
P(0,0, B)=b_{s s}=\frac{1-\gamma}{2-\gamma-\beta}, & P(1,0, B)=\varepsilon_{B}\left[(1-\gamma) g_{s s}+\beta b_{s s}\right]
\end{array}
$$

Further, we note that the probability of bit loss in the GOOD state $\varepsilon_{G}$ can be measured in terms of the average received signal-to-noise ratio in the GOOD state $S N R_{G}$, the utilized modulation scheme, and the number of transmit/receive antennas. For example utilizing BPSK modulation in a single transmit single receive antenna environment, we have

$$
\varepsilon_{G}=0.5\left\{1-\sqrt{S N R_{G} /\left(1+S N R_{G}\right)}\right\}
$$

Similarly, the the probability of bit loss in the BAD state $\varepsilon_{B}$ can be measured. Utilizing Equation (1) along with Equation (2) and Equation (3), the probability of a block loss is given by

$$
\Psi\left(n, t_{c}, \varepsilon_{G}, \varepsilon_{B}, \gamma, \beta\right)=1-\sum_{i=n-t_{c}}^{n} P(n, i)
$$

\section{BIT ERRor CORRECTION}

In this section, we discuss the protection of the source coding bits in a packet set associated with a progressive bitstream. We propose the use of rate compatible punctured RS error correction codes in each packet. The main goal of this section is to introduce an optimization framework that minimizes the expected distortion of the reconstructed image due to random bit errors in a single round of transmission.

\section{A. Optimization Formulation}

We assume the original size of the bitstream is $B S_{R}$ and so far $B S_{A}$ bits of the bitstream have been delivered. Hence, the remaining number of the bits in the bitstream have to be packetized into $N$ fixed-length packets with length $L$. The choice of packet length $L$ has to avoid segmentation in the data link and network layer protocols in order to preserve the effectiveness of channel coding operation at the bit level. Once the packet length $L$ is chosen, the number of packets $N$ for the first round is selected such that the collection of packets contains a number of source coding bits less than or equal $B S_{R}-B S_{A}$. We set $N=\min \left(\left\lfloor\alpha \frac{B_{T}}{L}\right\rfloor,\left\lfloor\frac{B S_{R}-B S_{A}}{L}\right\rfloor\right)$ where the parameters are defined in Section II. Denoting $R_{i}$ and $C_{i}$ respectively as the source and the channel coding bits associated with packet $i$ for $i \in\{1, \cdots, N\}$, we observe that $R_{i}+C_{i}=L$. We recall that 
utilizing our proposed channel coding scheme introduces a channel code rate of $r_{i}=\frac{R_{i}}{L}$ for packet $i$. The optimization problem is aimed at finding the parity assignment of each packet $C_{i}$ such that a measurement of the expected distortion is minimized. The expected distortion can be calculated as the probabilistic average of distortions associated with recovering the first $i-1$ packets in a given packet set and failing to recover packet $i$ with $i \in\{1, \cdots, N+1\}$ as

$$
\mathcal{E}[D]=D_{0} \Psi_{1}+\sum_{i=2}^{N+1} \Psi_{i} D_{i-1} \prod_{j=1}^{i-1}\left(1-\Psi_{j}\right)
$$

In Equation (7), $\Psi_{i}$ with $i \in\{1, \cdots, N\}$ is the failure probability of recovering packet $i, \Psi_{N+1} \triangleq$ $1, D_{i}$ with $i \geq 1$ is the distortion of a reconstructed image with the first $i$ packets, and $D_{0}=$ $\sigma^{2}$ is the source variance. The distortion $D_{i}$ is a function of the aggregate receiving rate $b_{i}=$ $\sum_{j=1}^{i} R_{j}$ and can be approximated by utilizing data fitting techniques. In [1], the authors propose an approximation of the rate-distortion function in the form of

$$
D_{i}\left(b_{i}\right)=\sum_{j=1}^{4} h_{j} e^{-l_{j} b_{i}}
$$

where $h_{j}$ and $l_{j}$ are parameters that are identified independently for different classes of images. Further, the probabilities $\Psi_{i}$ for $i=\{1, \cdots, N\}$ can be calculated from Equation (6) by setting $n=L$ and $t_{c}=\left\lfloor\frac{C_{i}}{2}\right\rfloor$. Making note that for the fixed-length packet $i$ specifying the data rate $R_{i}$ is equivalent to specifying the parity rate $C_{i}$, the optimization problem of the first round is expressed as

$$
\begin{array}{cl}
\underset{C_{1}, \cdots, C_{N}}{\min } & \mathcal{E}[D] \\
\text { Subject To: } & \sum_{i=1}^{N}\left(L-C_{i}\right) \leq B S_{R}-B S_{A} \\
& 0 \leq C_{i}<L, \quad i \in\{1, \cdots, N\}
\end{array}
$$

While Constraint (10) shows that the number of source coding bits in the $N$ packets adds up to no more than the number of bits in the remaining part of bitstream, Constraint (11) places a lower and an upper bound on the per packet channel coding bits. We note that the problem of (9) is subject to discrete constraints applied to available channel coding variables $C_{1}, \cdots, C_{N}$. It is hence categorized under NonLinear Programming (MINLP). Under the assumption of feasibility, the solution to the standard problem can provide a close estimate of the MINLP solution. Assuming the solution to the optimization problem of the first round specifies a per packet set of channel coding bits $\left\{C_{1}, \cdots, C_{N}\right\}$ and source coding bits $\left\{L-C_{1}, \cdots, L-C_{N}\right\}$, the optimization problem of the rounds beyond the first round are specified with the same cost function as Equation (7) for the per packet source coding bits of the first round but a different number of channel coding bits, $\Psi_{i}=0$ for previously delivered packets, and the constraint set below.

$$
\sum_{i \in \mathcal{F}}\left(C_{i}^{\prime}-C_{i}\right) \leq N L ; \quad C_{i}^{\prime}=C_{i}, \quad i \in \mathcal{R} ; \quad C_{i} \leq C_{i}^{\prime} \leq L_{\max }-\left(L-C_{i}\right), \quad i \in \mathcal{F}(12)
$$

where $N=\min \left(N_{F},\left\lfloor\alpha \frac{B_{T}}{L}\right\rfloor\right), L_{\text {max }}$ is the mother code length, $\mathcal{F}$ and $\mathcal{R}$ indicate the set of failed and recovered packets, $C_{i}$ and $C_{i}^{\prime}$ with $i \in \mathcal{F} \cup \mathcal{R}$ denote the current and the previous collective number of parity bits for packet $i$. While the formulations of this section specify a distortion-optimal problem, it can be easily changed to reflect a rate-optimal problem. 


\section{B. Optimization Solution}

In this section, we provide a discussion of solving the optimization problems of Section IV-A. Relying on the Lagrangian theory [3], we convert the problem of the first round to an optimization problem without constraints. The unconstrained minimization problem is defined as

$$
\min _{\Omega} L G_{D}=\min _{\Omega}\left\{\mathcal{E}[D]+\lambda\left(\sum_{i=1}^{N} C_{i}-N L+B S_{R}-B S_{A}\right)+\sum_{i=1}^{N} \mu_{i}\left(C_{i}-L\right)\right\}
$$

where $\Omega=\left\{C_{1}, \cdots, C_{N}\right\}$ and the parameters $\lambda, \mu_{1}, \cdots, \mu_{N}$ are the Lagrange multipliers.

Taking into consideration the discrete nature of our problem and considering the fact that (9), (10), and (11) are convex, we propose deploying Sequential Quadratic Programming (SQP) technique to solve the problem. In SQP, the necessary conditions for optimality are represented by Karush-KuhnTucker (KKT) conditions described below.

$$
\begin{aligned}
& \nabla L G_{D}\left(\Omega^{*}\right)=\left[\frac{\partial L G_{D}}{\partial C_{1}^{*}}, \cdots, \frac{\partial L G_{D}}{\partial C_{N}^{*}}, \frac{\partial L G_{D}}{\partial \lambda^{*}}, \frac{\partial L G_{D}}{\partial \mu_{1}^{*}}, \cdots, \frac{\partial L G_{D}}{\partial \mu_{N}^{*}}\right]=0 \\
& \lambda^{*}\left(\sum_{i=1}^{N} C_{i}^{*}-N L+B S_{R}-B S_{A}\right)=0 ; \quad \mu_{i}^{*}\left(C_{i}^{*}-L\right)=0 ; \quad \lambda^{*}, \mu_{i}^{*} \geq 0
\end{aligned}
$$

Further, $\lambda^{*}, \mu_{i}^{*} \geq 0$ for $i=1, \cdots, N$ if associated with an active inequality at the optimal point $\Omega^{*}$, i.e.,

$$
\left\{\begin{array} { l l l } 
{ \lambda ^ { * } \geq 0 } & { : } & { \text { if } \sum _ { i = 1 } ^ { N } C _ { i } ^ { * } = N L - B S _ { A } } \\
{ \lambda ^ { * } = 0 } & { : } & { \text { otherwise } }
\end{array} \quad \left\{\begin{array}{ll}
\mu_{i}^{*} \geq 0 & : \text { if } C_{i}^{*}=L \\
\mu_{i}^{*}=0 & : \text { otherwise }
\end{array}\right.\right.
$$

A variant of the quasi-Newton method [15] can then be used to iteratively find the solution to the optimization problem. We note that utilizing a variant of the quasi-Newton method is equivalent to solving a quadratic estimation of the problem in every iteration. The time complexity of solving the optimization problem is $\mathcal{O}(I d \log d)$ where $I$ indicates the number of iterations and $d$ indicates the degree of the overall quadratic estimation. The solution to the optimization problem of the second round and beyond is similar and is skipped here. We have observed that an average of ten and no more than twelve iterations are required for the convergence of our proposed optimization algorithm of the first round. The associated numbers for the algorithm of the second round and beyond depend on the number of lost packets but are generally smaller than the ones in the first round. Hence, the complexity results are quite good compared to other recursive optimization approaches such as dynamic programming introducing a time complexity in the order of $\mathcal{O}\left(d^{2}\right)$.

\section{Statistical Recovery from Packet ERAsure}

We now turn our focus on the statistical recovery of the packets associated with the bitstream of a progressive source coder in an erasure channel. We assume that individual packets of the set contain both source coding and/or parity bits the collection of which is treated as data for the purpose of packet erasure compensation. Similar to what was discussed in the previous section, we propose utilizing rate compatible punctured RS codes at the packet level to compensate for packet erasures. However, our approach in this section relies on providing a statistical guarantee for delivering a packet set. The term statistical guarantee of the packet set delivery is used to indicate that a packet set can be successfully delivered with a probability better than a given threshold assuming the specifications of the packet erasure channel are known. Our earlier work of [23] introduces the following algebraic placement algorithm with a time complexity of $\mathcal{O}(z k)$ to calculate the smallest number of required transmitted packets $u=k+z$ in order to guarantee the receipt of at least $k$ packets with a probability $\Pi$ or better for a system governed by the Gilbert or Gilbert-Elliott loss models.

\section{Statistical Guarantee for Packet Delivery Algorithm}


- Initialize $D(k, k)=\gamma^{k} \frac{1-\beta}{2-\gamma-\beta}+\gamma^{k-1}(1-\beta) \frac{1-\gamma}{2-\gamma-\beta}$

- for $(z=1$ to $k)\{$

- Calculate $P(k+z, k)$

- Update $D(k+z, k)=D(k+z-1, k)+P(k+z, k)$

- If $D(k+z, k) \geq \Pi \quad$ Break

\} $/ *$ for $(z=1$ to $k) * /$

- Report the number of required packets, $n=k+z$

The quantities of interest in the algorithm above are described as follows. $P(k+z, k)$ the probability of receiving $k$ packets from $k+z$ transmitted packets is given by Equation (1). The recursive equations (2) and (3) are utilized to calculate $P(k+z, k)$. We note that in the case of capturing packet erasure the Gilbert loss model can be utilized. It is a special case of the Gilbert-Elliott model with $\varepsilon_{G}=0$ and $\varepsilon_{B}=1$. The closed form probability of $P(k+z, k)$ under the Gilbert model can also be found in [23]. Further, $D(k+z, k)$ the probability of receiving $k$ packets or more from $k+z$ transmitted packets is defined as

$$
D(k+z, k)=\sum_{i=k}^{k+z} P(k+i, i)
$$

\section{Numerical ANALysis}

In this section, we present our simulation results based on the protocol of Section II and the discussion of the sections following it. For our simulation, we consider the transmission of a SPIHT [14] encoded $512 \times 512 \times 8 b p p$ gray scale Lena image over a channel characterized by correlated loss. We utilize Gilbert-Elliott and Gilbert loss models to describe the bit errors and packet erasures, respectively. We set the transition probabilities of both models as $\gamma=0.99873$ and $\beta=0.875$ corresponding to an average burst length of 8 . Besides the trivial choice of error probabilities in the case of Gilbert model, per state error probabilities of the Gilbert-Elliott model are calculated based on Equation (5) with $S N R_{G}=10 S N R_{B}$. In our experiments, we use rate compatible punctured RS codes with a maximum length 255 over $G F(256)$. Further, we set $\alpha=0.2, B_{T}=512 \times 512$, and $B S_{R}=512 \times 512$ for a compression ratio of 8 . For our statistical packet delivery algorithm, we select $\Pi=0.95$. The set of parameters associated with the Lena image in Equation (8) are set as $\left(h_{1}, h_{2}, h_{3}, h_{4}, l_{1}, l_{2}, l_{3}, l_{4}\right)=(1276.7,117.2,26.9,279.1,331.8472,11.2685,1.5792,50.2822)$.

Fig. 2 shows the plots of $P S N R=10 \log _{10} \frac{255^{2}}{\mathcal{E}[D]}$ versus $S N R_{G}$ for three different values of the packet length $L$. Our choices of packet lengths guarantee that there is no segmentation/reassembly of UDP or ATM packets over Ethernet and/or IEEE 802.11 frames. For a given packet size, the plots describe how the quality of the reconstructed image improves as the average signal-to-noise ratio increases. Every point in the curves indicates an average value taken over 5 simulations. The plots show a similar pattern with different choices of the packet size. The quality of the reconstructed image remains at $P S N R=14.53 d B$ for any choice of $S N R_{G}$ below a low threshold. The threshold moves from $S N R_{G}=3 d B$ in the case of $L=100$ Bytes to $S N R_{G}=9 d B$ in the case of $L=200$ Bytes. For our given budget of $B_{T}=512 \times 512$, increasing $S N R_{G}$ beyond the low threshold improves the quality of the reconstructed image until reaching a high threshold. The average value of the high threshold is at $P S N R=34.2 \mathrm{~dB}$. The quality of the reconstructed image remains near perfect for any choice of $S N R_{G}$ beyond the high threshold. Assuming availablity of transmission budget, the quality of the reconstructed image can improve to $39.4 d B$ at an ideal case not shown here. The high threshold moves from $S N R_{G}=7 d B$ in the case of $L=100$ Bytes to $S N R_{G}=16.8 d B$ in the case of $L=200 B y t e s$. Fig. 3 shows sample images corresponding to the low, medium, and high signal-to-noise ratio regions in Fig. 2 for the choice of $L=100$ Bytes. 


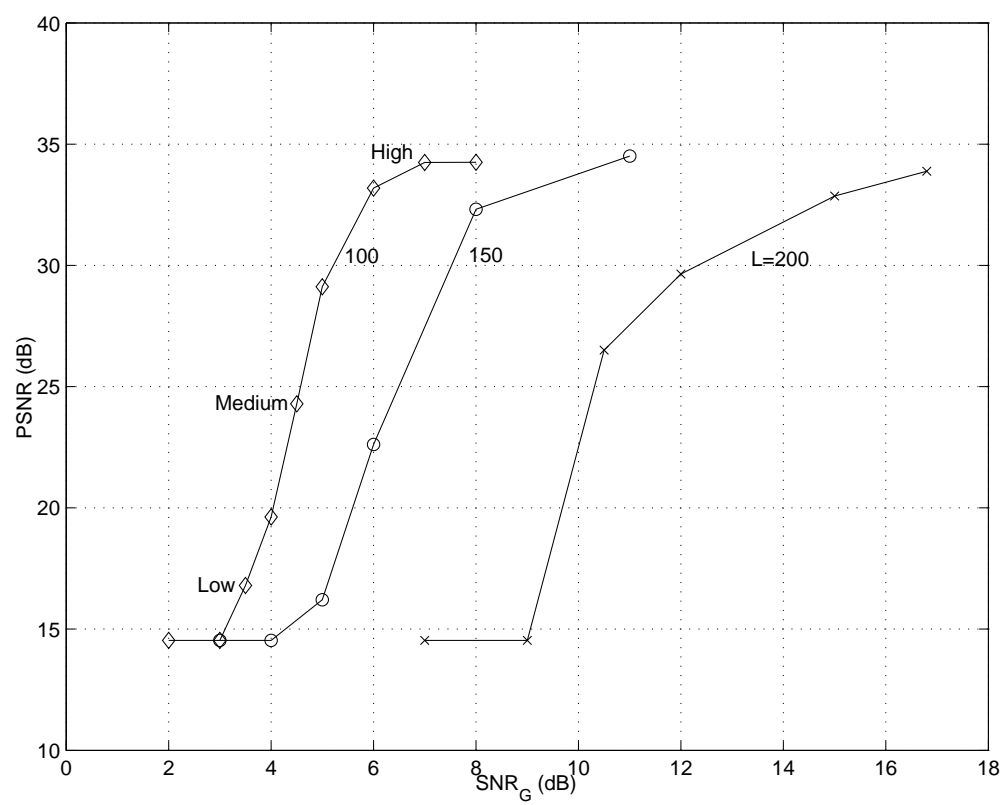

Fig. 2. Plots of $P S N R$ versus $S N R_{G}$ for different choices of the packet size.

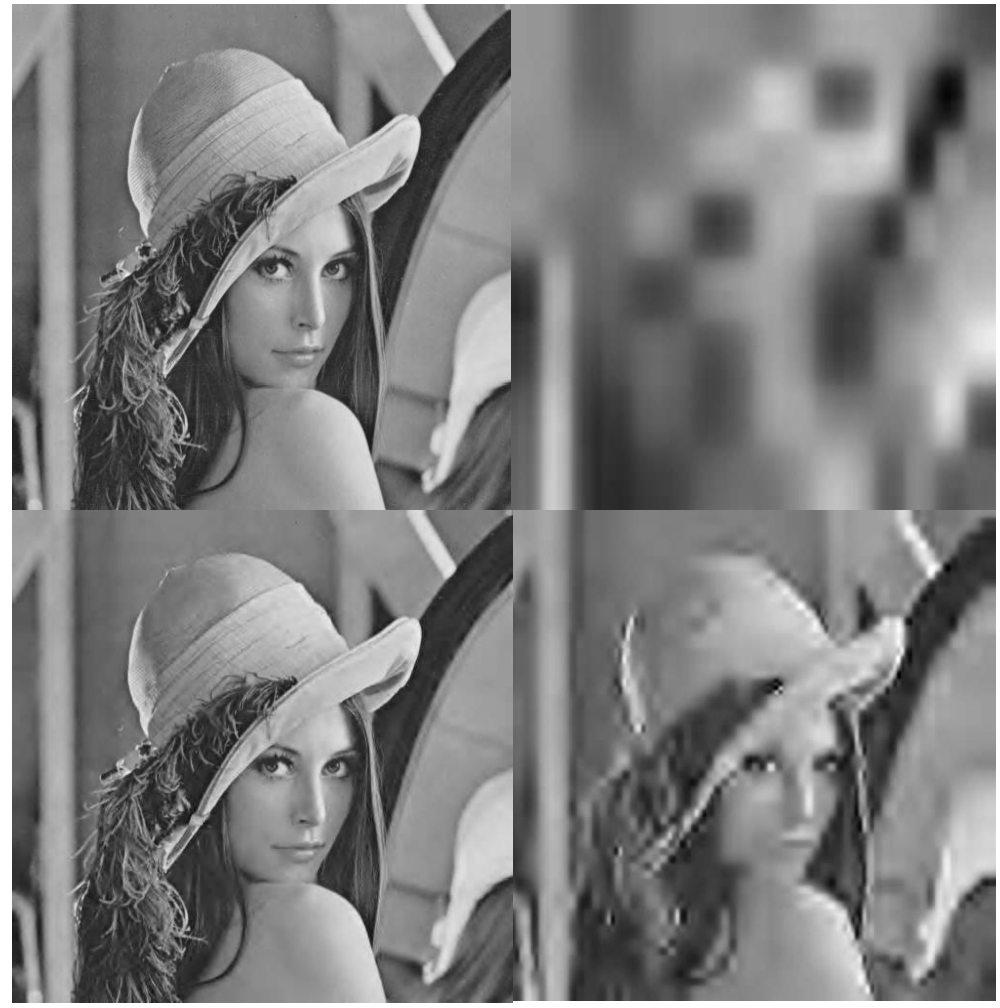

Fig. 3. A comparison of the original and reconstructed sample Lena images for three different values of $S N R_{G}$ using $L=100 B y t e s$. Clockwise from the top left: the original image, the reconstructed image at $S N R_{G}$ equal to $3.5 d B$, $4.5 d B$, and $7 d B$, respectively. 


\section{CONCLUSIONS}

In this paper, we presented a statistical optimization framework for progressive transmission of images over noisy channels characterized by temporally correlated loss. Relying on rate compatible punctured RS codes, our framework was able to compensate for random bit errors as well as packet erasures. We considered the impacts of transmission over channels with memory represented by the Gilbert-Elliott model. In order to cope with random bit errors, we introduced an optimization framework to minimize the expected distortion of a reconstructed image. We recursively solved our optimization problem with a relatively low time complexity. Next, we provided an algorithm that was capable of statistically compensating for packet erasures. Relying on the receiver feedback, we integrated our bit error and packet erasure results in the form of a type II hybrid FEC-ARQ algorithm. Finally, we numerically validated our results. We are currently in process of optimizing the bandwidth allocation between the two components of our framework. Further, we are extending our work to one-to-many transmission scenarios.

\section{REFERENCES}

[1] A. Appadwedula, D.L. Jones, K. Ramchandran, I. Konzintsev, "Joint Source Channel Matching for A Wireless Communications Link," In Proc. of IEEE ICC, 1998.

[2] B.A. Banister, B. Belzer, T.R. Fischer, “Robust Image Transmission Using JPEG2000 and Turbo-Codes,” IEEE Sig. Proc. Letters, April 2002.

[3] D.P. Bertsekas, "Nonlinear Programming, 2nd Edition," Athena Scientific Publishing, 1999.

[4] R. E. Blahut, "Algebraic Codes for Data Transmission," Cambridge University Press, 2003.

[5] V. Chande, H. Jafarkhani, N. Farvardin, "Image Communication over Noisy Channels with Feedback," In Proc. of IEEE ICIP, 1999.

[6] V. Chande, N. Farvardin, "Progressive Transmission of Images over Memoryless Channels," IEEE JSAC, June 2000.

[7] P.C. Cosman, J.K. Rogers, P.G. Sherwood, K. Zeger, "Combined Forward Error Control and Packetized Zerotree Wavelet Encoding for Transmission of Images over Varying Channels,” IEEE Trans. Image Proc., June 2000.

[8] E.O. Elliott, "Estimates on Error Rates for Codes on Burst-Noise Channels," Bell Syst. Tech. J., Sept. 1963.

[9] H. Jafarkhani, P. Ligdas, N. Farvardin, "Adaptive Rate Allocation in a Joint Source-Channel Coding Framework for Wireless Channels," In Proc. of IEEE VTC, April 1996.

[10] J. Lu, A. Nosratinia, B. Aazhang, "Progressive Source-Channel Coding of Images over Bursty Error Channels," In Proc. of IEEE ICIP, 1998.

[11] H.S. Malavar, “Fast Progressive Wavelet Coding,” In Proc. of IEEE DCC, 1999.

[12] D.M. Mandelbaum, “An Adaptive Feedback Coding Scheme Using Incremental Redundnacy,” IEEE Trans. Inform. Theory, May 1974.

[13] E. Ordentlich, M.J. Weinberger, G. Seeroussi, "A Low Complexity Modeling Approach for Embedded Coding of Wavelet Coefficients," In Proc. of IEEE DCC, 1998.

[14] A. Said, W.A. Pearlman, "A New Fast and Efficient Image Codec Based on Set Partitioning in Hierarchical Trees," IEEE Trans. Circuits and Syst. for Video Technology, June 1996.

[15] D.F. Shanno, "Conditioning of Quasi-Newton Methods for Function Minimization,” Mathematics of Computing, Vol. 24, pp 647-656, 1970.

[16] J.M. Shapiro, "Embedded Image Coding Using Zerotrees of Wavelet Coefficients," IEEE Trans. Sig. Proc., Dec. 1993.

[17] P.G. Sherwood, K. Zeger, "Progressive Image Coding for Noisy Channels,” IEEE Sig. Proc. Letters, July 1997.

[18] B.S. Srinivas, R. Ladner, M. Azizoglu, E.A. Riskin, "Progressive Transmission of Images Using MAP Detection over Channels with Memory," IEEE Trans. Image Proc., April 1999.

[19] V. Stankovic, R. Hamzaoui, D. Saupe, "Fast Algorithm for Rate-Based Optimal Error Protection of Embedded Codes," IEEE Trans. Commun., Nov. 2003.

[20] D. Taubman, M. Marcellin, "JPEG2000: Image Compression Fundamentals, Standards, and Practice," Kluwer, 2001.

[21] D. Taubman, A. Zakhor, "Multi-Rate 3-D Subband Coding of Video,” IEEE Trans. Image Proc., Sept. 1994.

[22] S. Wicker, "Error Control Systems for Digital Communications and Storage," Prentice-Hall, 1995.

[23] H. Yousefi'zadeh, H. Jafarkhani, "Statistical Guarantee of QoS in Communication Networks with Temporally Correlated Loss ,” In Proc. of IEEE GLOBECOM, 2003. 